\title{
Estado del arte en detección de fugas y propuesta de máquina de soporte vectorial para el análisis de estanqueidad en envases ${ }^{1}$ \\ State of the art in leak detection and support vector ma- chine for the analysis of sealing in containers
}

Estado da arte em movimento de vazamento de detecção e sup-
port vector machine para a análise de vedação em embalagens

Recibido: octubre de 2012

Aprobado: diciembre de 2012
Luis Francisco Niño Sierra ${ }^{2}$

Darío Amaya Hurtado ${ }^{3}$

Mauricio Mauledoux Monroy ${ }^{4}$

\section{Resumen}

El presente artículo es una recopilación de los métodos más utilizados en análisis de estanqueidad o detección de fugas, como antecedentes de la realización de un sistema que aplica la inteligencia artificial para este tipo de análisis. Se presenta también la propuesta de usar una máquina de soporte vectorial en este sistema.

Palabras clave: análisis de estanqueidad, detección de fugas, máquina de soporte vectorial.

\begin{abstract}
This article is a compilation of the analysis methods used in sealing or leak detection, as background to the implementation of a system that applies artificial intelligence for this type of analysis. We also present the proposal of using a support vector machine in this system.
\end{abstract}

Keywords: analysis of sealing, leak detection, support vector machine.

\section{Resumo}

Este artigo é uma visão geral dos métodos de análise utilizados na detecção de vedação e vazamento, como pano de fundo para a implementação de um sistema de inteligência artificial que se aplica a este tipo de análise. Também apresenta a proposta de utilização de uma máquina de vetor de suporte neste sistema.

Palavras-chave: análise de vedação, detecção de vazamento, máquinas de vetores de suporte.

1 Artículo de Revisión.

2 Docente de la Universidad Distrital Francisco José de Caldas, Bogotá - Colombia. Contacto: Ifninos@udistrital.edu.co

3 Docente de la Universidad Militar Nueva Granada, Bogotá - Colombia. Contacto: dario.amaya@unimilitar.edu.co

4 Docente de la Universidad Militar Nueva Granada, Bogotá - Colombia. Contacto: mauricio.mauledoux@unimilitar.edu.co 


\section{Introducción}

La detección de fugas ha sido un área de gran interés en el sector industrial, en especial en los sistemas de transporte de petróleo y de gas, por los costos ambientales, sociales y económicos que implican para una región o país. Es así como en todo el mundo existen normas para la explotación y distribución de combustibles y multas económicas muy elevadas en los casos en los que se incumplen. En tuberías hay muchos métodos empleados para la detección de fugas, que pueden ser clasificados en métodos directos e indirectos (Chen, Shi, Zhang, et al., 2003; Wang, Jianguo, Weidong, et al., 2002). En otras áreas también se han desarrollado sistemas o métodos de detección de fugas, como es el caso de tuberías de transporte de agua, pruebas en diferentes tipos de envases y pruebas en sistemas microelectromecánicos o MEMS por sus siglas en inglés.

Muchas herramientas o métodos se han aplicado, hasta el punto que en la actualidad los algoritmos de inteligencia artificial están liderando esta tarea con excelentes resultados.

\section{Métodos acústicos}

La adquisición de señales acústicas con fin de realizar la detección de fugas en tuberías ha sido de gran importancia; sin embargo, la aparición de señales acústicas que no provienen de fugas reales genera inconvenientes en estos métodos de detección (Rongsheng, Gongtian, \& Shifeng, 2002). Las emisiones acústicas pueden ser definidas como una onda elástica transitoria, generada por la rápida liberación de energía dentro del material (Rongsheng, Gongtian, \& Shifeng, 2002). El análisis de estas emisiones acústicas permite la identificación de fugas, incluso a distancias lejanas. Las técnicas y equipos desarrollados basados en la señal acústica han sido efectivos en tuberías de agua (Jin, Yumei, \& Ping, 2010). Algunos instrumentos convencionales empleados en la detección de fugas son: varillas equipadas con auriculares, geófonos y micrófonos (Golby \& Woodward, 1999; Hunaidi, Wang, Bracken, Gambino, \& Fricke, 2004)); sin embargo, su efectividad depende, en gran medida, de la habilidad del operario. Con el fin de evitar la dependencia del usuario, los métodos que emplean procesamiento digital de señales acústicas han sido de gran ayuda y han hecho que las diferentes técnicas tengan avances importantes en cuanto a su efectividad.

Los instrumentos desarrollados a partir de esta idea están conformados en general de la siguiente manera:

- Un sensor o conjunto de sensores se emplean para explorar la tubería.

- Las señales allí obtenidas son transmitidas mediante sistemas de adquisición de datos, que incluyen filtros y amplificadores, a sistemas de procesamiento de información como microcontroladores, PC o DSP.

- Por último, una unidad de procesamiento se encarga de la identificación de las fugas; allí se puede emplear una de muchas herramientas de procesamiento disponibles; en general, a esta tarea puede dedicarse un solo algoritmo o la combinación de algunos de ellos. En Jiao, Qingxin, Guanghai, \& Jingyan (2006), un algoritmo o modelo de procesamiento se encarga de la extracción de las características de las señales y otro, a partir de estas características, se encarga de la identificación de las fugas correspondientes.

Los métodos acústicos son empleados ampliamente en tuberías que transportan gas, petróleo o incluso agua.

\section{Presión diferencial}

Los métodos de detección de fugas mediante la medición de presión diferencial se han empleado principalmente en el control de calidad en la fabricación de envases. En este caso se trata de la inyección de aire en el envase bajo prueba, hasta obtener una presión determinada, y luego analizar el comportamiento de la señal de presión medida entre el interior del envase y un punto externo, en el cual la presión es constante o plenamente conocida. La gran mayoría de los equipos desarrollados para esta tarea basan su funcionamiento en la simple 
comparación, durante un lapso determinado, de la señal con respecto a un valor fijo o umbral definido. Este valor de umbral se establece después de una gran cantidad de pruebas, que permiten determinar el valor óptimo para cada aplicación.

El método es simple; sin embargo, permite el empleo de equipos que no cuentan con poderosas herramientas de procesamiento, como es el caso del PLC, el cual es ampliamente usado a nivel industrial. Sin embargo, las limitantes de este método son evidentes cuando se trata de microfugas, ya que en estos casos la presión dentro del envase cambia muy lentamente, y durante el periodo de comparación no alcanza a superar el umbral, lo que da un resultado erróneo. Algunas compañías han usado este método por mucho tiempo, mejorando la técnica hasta lograr resultados muy buenos en función del diámetro de la fuga capaz de detectar (Bonfiglioli Engineering S.p.A.), incluso algunas de estas compañías anuncian en la actualidad el uso de inteligencia artificial para mejorar la precisión y la capacidad de detección. Sin embargo, estos desarrollos se mantienen ocultos dado el carácter comercial de estas compañías. Una variante de este método consiste en la producción de una pequeña deformación en el envase cuando se tiene presurizado; el análisis de la relación presión-deformación permite determinar si se trata de un elemento con una fuga o no (Bhattacharyya \& Johnson, 1977). Aun así, no se conoce si este método se emplea en la actualidad por los fabricantes de las máquinas de pruebaenvases.

\section{Métodos de procesamiento utilizando inteligencia artificial en la detección de fugas}

Como se mencionó, los métodos tradicionales, acústicos o de presión diferencial, por sí solos no son suficientes para garantizar la calidad de un envase o si, por el contrario, se presenta una fuga en alguna zona determinada del mismo. En la actualidad las técnicas de procesamiento digital de señales, con las modernas herramientas proporcionadas por la inteligencia artificial, son empleadas en casi todas aplicaciones de análisis de señales; la detección de fugas no es la excepción. Una breve descripción de algunas de estas herramientas se presenta a continuación:

\section{Autocorrelación}

La principal propiedad de la autocorrelación es la de entregar una medida de la coherencia de una serie en el dominio del tiempo. Esta propiedad en realidad lo que permite es tener una idea de la autosimilitud de la señal (Jin, Yumei, \& Ping, 2010). Algunas de las características que se pueden obtener a partir de la autocorrelación son (Haykin, 1998): la rapidez con la que una señal cambia con respecto al tiempo, dónde un proceso tiene una componente periódica, y si un valor en un instante es similar o no con valores anteriores. En Jin, Yumei, \& Ping (2010) las características de autosimilitud obtenidas por medio de la autocorrelación son usadas en la capa de entrada de una red neuronal, que se encarga de determinar la existencia o no de una fuga en una red de distribución de agua.

\section{Redes neuronales}

Una red neuronal puede definirse como una estructura o sistema construido para hacer uso de los principios organizacionales del cerebro humano. Los elementos fundamentales de esta red se conocen como neuronas artificiales, las cuales cuentan con alta conectividad entre ellas y con la capacidad de realizar operaciones de cálculo (Avelino et al., 2009). Las redes neuronales artificiales son especialmente usadas en el reconocimiento y clasificación de patrones, en la compresión de imágenes y en el filtrado de señales. Como se mencionó, una red neuronal artificial es usada en la detección de fugas en tuberías de agua. Así mismo, se usan redes neuronales para la detección de fugas en tuberías de petróleo (Ming \& Wei-qiang, 2006). En este caso, el algoritmo para la detección de la fuga parte de la adquisición de señales por medio de sensores de presión; estas señales pasan por una etapa de adecuación, luego por medio de wavelets se realiza la descomposición de las señales y por último mediante la red neuronal se extraen las características y se clasifican los perfiles de fuga. 


\section{Wavelets (onditas)}

La transformada de onditas, o wavelets, es considerada un tipo especial de transformada de Fourier, que permite obtener información en función del tiempo y de la frecuencia de forma cuasi simultánea. La transformada de onditas es muy utilizada en procesamiento de señales, es común su aplicación en filtros, compresión y descompresión de datos y en extracción de características. Esta última aplicación es empleada en Ming\& Wei-qiang (2006) como etapa previa a la red neuronal artificial, que se encarga de la clasificación de estas características para determinar la presencia de la fuga. Así mismo, en Zadeh (1965) se emplean wavelets en la detección de fugas de manera inmediata en tuberías de petróleo, con el fin de evitar robo, minimizar pérdidas y garantizar el normal funcionamiento del sistema de transporte. En este caso se usan dos sensores de presión en los extremos del tramo de tubería que se desea explorar; cuando una fuga se produce, genera una onda de presión que se transmite a lo largo de la tubería. Esta onda representa una caída de presión que se conoce como punto de rareza de la señal (Zadeh, 1965). El algoritmo de wavelet permite detectar este punto de rareza y el momento en que se produce.

\section{Teoría del control difuso}

La lógica difusa es una de las herramientas que han encontrado aplicaciones en áreas como la economía, la medicina, la administración y los sistemas de control, entre otras. Esta teoría, que fue introducida por Zadeh (1965), crea un nuevo tipo de variables, las variables difusas, que son descritas por expresiones lingüísticas como largo, alto o rápido, etc. Todo un proceso matemático alrededor de estas nuevas variables se ha desarrollado y ha permitido dar solución a problemas muy difíciles de representar con las variables tradicionales. En Tongtai (1997) se emplea la teoría de control difuso para la detección de la zona de fuga antes de que se produzca la perforación. La mayoría de los métodos empleados analizan la información obtenida después de producirse la fuga, con los correspondientes riesgos o pérdidas que esto implica (Tani et al., 2006). Por lo anterior, este método es aplicado en la prevención y predicción de fugas.

\section{Teoría del caos}

Como se ha visto hasta ahora, la detección de fugas en tuberías de transporte de petróleo, gas o agua ha tenido un gran interés en los investigadores. No menos importante es la detección de fugas en plantas refinadoras de petróleo, por el peligro que esto implica para el personal que allí trabaja. En Tani, Nagasako, \& Lokibe (2004) se plantea un sistema de detección de fugas de gas a alta presión en refinerías de petróleo, basado en la teoría del caos. El método consiste en aplicar el Criterio de Información de Caos (Vaz Junior, 2010) a señales acústicas adquiridas por medio de un micrófono. Como fuente sonora se usa un generador artificial de gas a alta presión, o simulador de la fuga, que se ubica a 16 metros del sensor. En estas condiciones, el micrófono captura no solamente el sonido del gas a alta presión, sino también los sonidos ambientales representados como ruido de fondo. Con base en estudios anteriores (Takens, 1981) se puede asumir que el ruido de fondo está conformado por elementos no determinísticos, mientras que las señales producidas por la fuga del gas a alta presión contienen componentes determinísticas. Con el fin de discriminar entre los dos tipos de señales adquiridas, se usa el método TPM (por las siglas de trajectory parallel measure) (Fujimoto \& Lokibe, 1999), con el cual se puede determinar la presencia de fuga comparando con un valor umbral fijado.

\section{Predicción ARX}

Otro de los algoritmos para la detección de fugas en tuberías está basado en un predictor autorregresivo MIMO (múltiples entradas / múltiples salidas) (Allister, 1988), en este caso con un filtro de entrada. Este algoritmo está conformado por dos etapas, una que se encarga de la detección de la fuga y otra de su localización en el tiempo. Para esto se almacena un vector de datos en una ventana de tiempo con la información necesaria para determinar el instante en que se produce la fuga.

\section{Estabilidad de Liapunov}

Un método basado en el criterio de estabilidad de Liapunov se usa para la detección de fugas. En 
Lawrence (1990) se presenta el trabajo realizado con pruebas en la tubería de una planta petrolera en Nigeria. El método consiste en tomar un modelo tradicional de flujo de líquidos en una tubería; se incluye un término que representa una fuga. Los diferentes criterios de estabilidad en sistemas de flujo fueron aplicados para desarrollar el modelo para la detección de la fuga. Se usa una matriz de estabilidad para determinar los eigenvalores del sistema. Una fuga es detectada cuando alguno de los valores propios es menor que -1. Mediante un programa de simulación, se determina la certeza de la fuga; en cada iteración del programa se evalúan los eigenvalores, asignando votos a favor o en contra de la presencia de la fuga. Por la teoría de probabilidad y la estadística (Gan et al., 2008) se aplica el criterio de Bayes. De acuerdo con Kingsley, Abhulimen, \& Susu (2004), cuando una fuga se produce en una tubería, la onda sonora que se genera viaja por el fluido a velocidad sónica. Con mediciones de tiempo de propagación de la onda, se puede llegar a obtener el tamaño de la fuga.

\section{Espectrometría de masa de helio e interferometría óptica}

Por último, en este recorrido por los métodos para detección de fugas se incluye una aplicación que ha brindado gran apoyo en los avances tecnológicos en todas las áreas de la vida humana: los MEMS o Sistemas Microelectromecánicos. Son muchos los dispositivos de este tipo, como sensores, acelerómetros, giróscopos, sensores de infrarrojo, que deben ser empacados al vacío y, más aún, el tiempo de vida depende de la duración de este vacío (Goswami \& Han, 2008). Cuando se trata de empaquetamientos pequeños, las exigencias de hermeticidad son mayores, dado que estos son susceptibles de microfugas. Estudios han mostrado que la tasa máxima de fugas en empaques pequeños es varios órdenes de magnitud menor que los empaques que son más grandes (Tao\& Malshe, 2005). La espectrometría de masa de helio se basa en el bombardeo de un elemento con helio presurizado por un tiempo determinado; luego se pasa a un espectrómetro, donde se mide la velocidad de salida del helio. Por otro lado, la interferometría óptica se basa en el patrón de interferencia obtenido al atacar el espécimen sometido a presión con un haz láser; el frente de onda reflejado produce un interferograma que es capturado con una cámara.

\section{Máquina de soporte vectorial}

Las máquinas de soporte vectorial (SVM por sus siglas en inglés) han sido ampliamente usadas en tareas de clasificación de información (Lin \& Chen, 2008); sin embargo, no se encuentran reportes de aplicaciones en la detección de fugas a pesar de su gran fortaleza a la hora de distinguir datos entre dos clases. La SVM es considerada una gran herramienta de clasificación lineal o no lineal (Burges, 1998) y ha sido empleada en problemas como ecualización de canal de ultrabanda ancha (Musbah \& Zhu, 2007), estimación de canal en sistemas de multiplexación de frecuencia ortogonal (Fernández-Getino \& Rojo, 2006), detección de actividad de voz (Fengyan, Changchun, \& Yan, 2004). Un clasificador SVM es básicamente un algoritmo que maximiza la distancia entre dos clases, minimizando error de clasificación. La SVM busca, a partir de un conjunto de datos de entrenamiento, un hiperplano que separe las dos clases a las que pertenecen. Este hiperplano puede ser tan simple como una recta en el caso de que se trate de datos linealmente separables, como el de la figura 1 , o puede estar compuesto por muchas fronteras

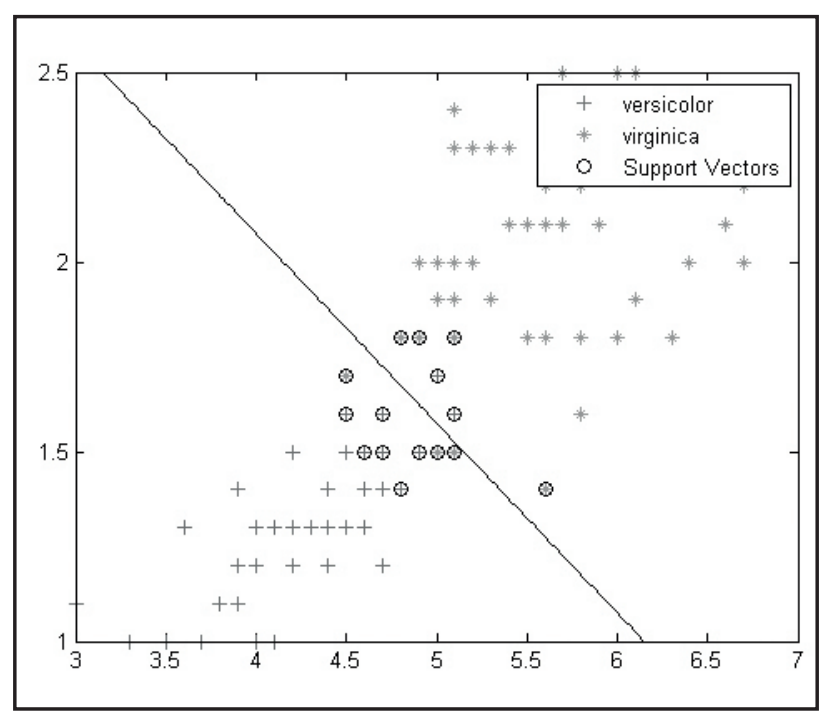

Figura 1. Datos linealmente separables.

Fuente: elaboración propia. 
de decisión que conforman un hiperplano más complejo, como es el caso de la figura 2.

En un sistema de detección de fugas en envases, la señal de presión obtenida entre el interior del envase y un punto de referencia exterior puede clasificarse en una de dos clases según se trate de un envase en perfecto estado o uno con una pequeña fuga. El proceso de detección se presenta en la figura 3. La mayoría de los detectores basan su funcionamiento en la comparación de esta señal con un valor umbral previamente establecido; en caso de que la señal decaiga por debajo de este nivel, se clasifica como defectuoso; en caso contrario se clasifica como bueno. Dadas estas características, se propone como herramienta de procesamiento una SVM para la realización de un sistema de análisis de fugas por presión diferencial. Los resultados obtenidos serán objeto de un segundo artículo.

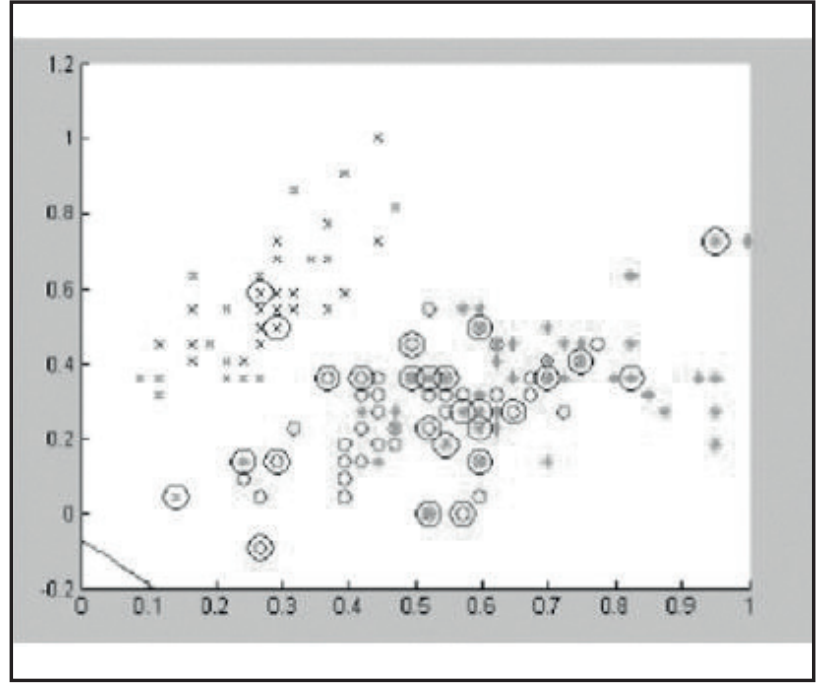

Figura 2. Datos para un clasificador no lineal.

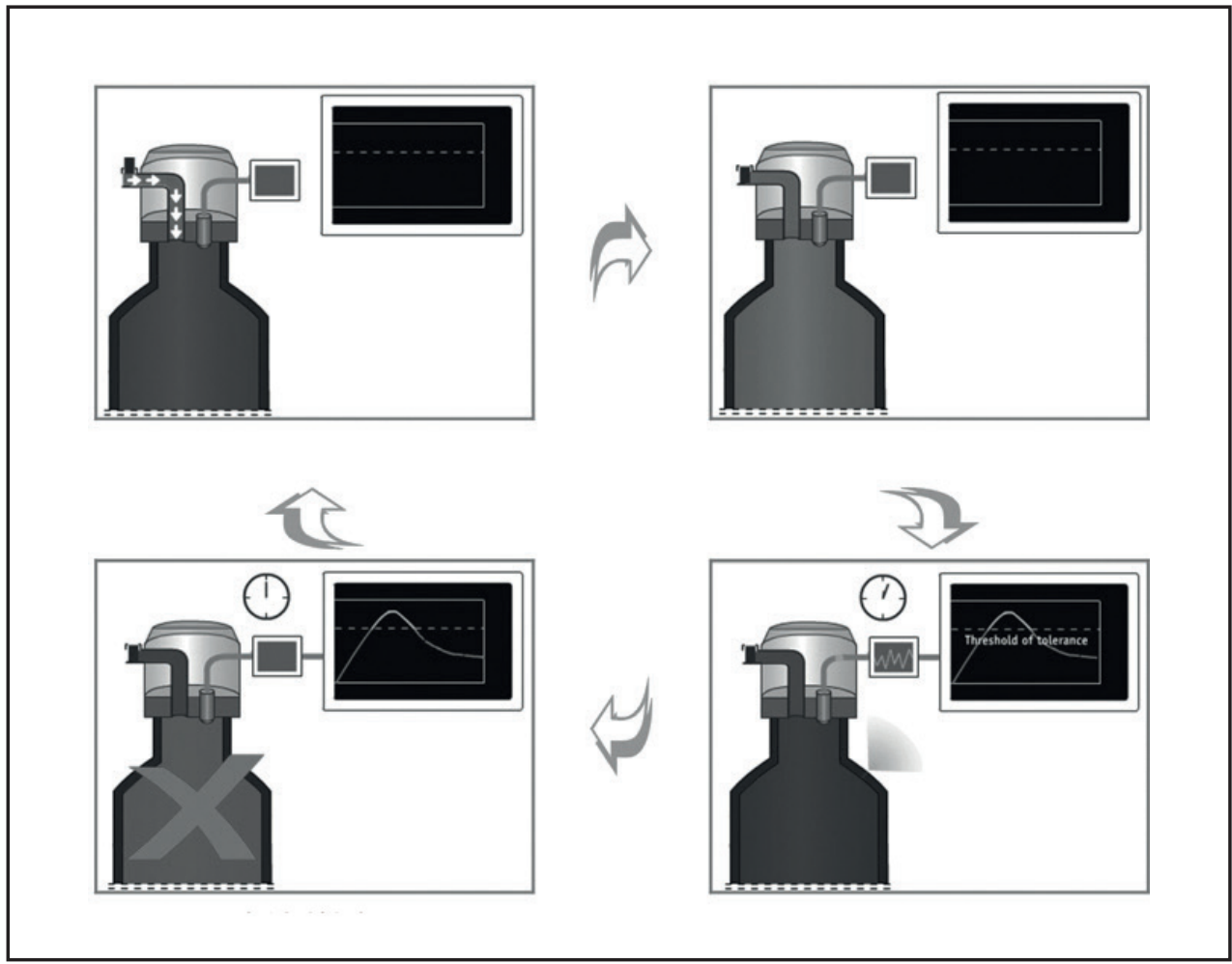

Figura 3. Proceso de detección de presión en una prueba de estanqueidad.

Fuente: http://www.pg-leak.com/en/container-leak-tester-01.html. 


\section{Referencias}

Allister, E. W. (1988). Pipeline rules of thump handbook: A manual for quick, accurate solutions to everyday pipeline problems. 2nd ed. Houston: Gulf Publishing House.

Avelino, Á. M., et al. (2009). Real time leak detection system applied to oil pipelines using sonic technology and neural networks. IEEE.

Batzias, F., Siontorou, C., \& Spanidis, P.-M. (2011). Designing a reliable leak bio-detection system for natural gas pipelines. Journal of Hazardous Materials, 186(1), 35-58.

Beck, S. M. B., Curren, M. D., Sims, N. D., \& Stanway, R. (2005). Pipeline network features and leak detection by cross-correlation analysis of reflected waves. Journal of Hydraulic Engineering, ASCE 131(8), 715-723.

Bhattacharyya, G. K. \& Johnson, R. A. (1977). Statistical concepts and methods. John Wiley \&Sons.

Billmann, L. \& Isermann, R. (1987). Leak detection methods for pipelines. Automatica, 23(3), 381-385.

Bimpas, M., Amditis, A., \& Uzunoglu, N. (2010). Detection of water leaks in supply pipes using continuous wave sensor operating at 2.45 GHz. Journal of Applied Geophysics 70, 226236.

Bonfiglioli Engineering S.p.A. Recuperado de www.bonfiglioliengineering.com

Burges Christopher, J. C. (1998). A tutorial on support vector machines for pattern recognition. Data Mining and Knowledge Discovery 2, 121167.

Chen, H., Shi, X., Zhang, Y., et al. (2003). The advancement of pipelines leak detection technology. Security and Environment Engineering, 10(3), 58-61.
Colombo, A. F., Lee, P., \& Karney, B. W. (2009). A selective literature review of transient-based leak detection methods. Journal of Hydro-environment Research, 2, 212-227.

Covas, D. \& Ramos, H. (1999). Leakage detection in single pipelines using pressure wave behaviour. In: Fifth International Conference on Computing and Control in the Water Industry (CCWI), Exeter, England, September 1999. Proceedings of CCWI'99, Exeter, UK, 287-299.

Covas, D., Ramos, H., Lopes, N., Almeida, A. B. (2006). Water pipe system diagnosis by transient pressure signals. In: Eighth Annual Water Distribution Systems Analysis Symposium, Cincinnati, $\mathrm{OH}$, August 27-30.

Da Silva, H. V., Morooka, C. K., Guilherme, I. R., da Fonseca, T., \& Mendes, J. R. P. (2005). Leak detection in petroleum pipelines using a fuzzy system. Journal of Petroleum Science and Engineering, 49, 223-238.

El-Shiekh, T. (2010). Leak detection methods in transmission pipelines. Energy Sources, Part A: Recovery, Utilization, and Environmental Effects, 32(8), 715-726.

Fengyan, Q., Changchun, B., \& Yan, L. (2004). A novel two-step SVM classifier for voiced/unvoiced/silence classification of speech. ISCSLP 2004, 77-80.

Fernández-Getino García, M. J. \& Rojo Álvarez, J. L. (2006). Support Vector Machines for robust channel estimation in OFDM. IEEE Signal Processing Letters, 13(7), 397-400.

Ferrante, M. \& Brunone, B. (2004). Pressure waves as a tool for leak detection in closed conduits. Urban Water Journal 1(2), 145-156.

Fujimoto, Y. \& Lokibe, T. (1999). Measurement of Determinism in Time Series by Chaotic Approach and its Applications. International Journal of Advanced Computational Intelligence, 3(1), 50-55. 
Golby, J. \& Woodward, T. (1999). Find that leak. IEE Review, 45, 219-221.

Goswami, A. \& Han, B. (2008). On ultra-fine leak detection of hermetic wafer level packages. IEEE.

Guo, X.-l., et al. (2012). Analysis of first transient pressure oscillation for leak detection in a single pipeline. Journal of Hydrodynamics, 24(3), 363-370.

Haykin, S. (1998). Neural networks: A comprehensive foundation. Prentice Hall. Recuperado de www.bonfiglioliengineering.com/machinery. php? sez $=6 \&$ cont $=24$

Hunaidi, O., Wang, A., Bracken, M., Gambino, T., \& Fricke, C. (2004). Acoustic methods for locating leaks in municipal water pipe networks. International Conference on Water Demand Management, Dead Sea, Jordan, May 30-June 3, pp. 1-14.

Jönsson, L. (2001). Experimental studies of leak detection using hydraulic transients. In: 29th IAHR Congress Proceedings, IAHR, Sept. 1621, Beijing, China.

Jourdain, A., et al. (2002). Investigation of the hermeticity of BCB-sealed cavities for housing (RF-) MEMS devices, in Proc. 15th IEEE Int. Conf. Micro Electro Mechanical Systems, Las Vegas, NV, Jan., pp. 677-680.

Kim, M. \& Lee, S. (2009). Detection of leak acoustic signal in buried gas pipe based on the timefrequency analysis. Journal of Loss Prevention in the Process Industries, 22, 990-994.

Kingsley, E., Abhulimen, A., \& Susu, A. (2004). Liquid pipeline leak detection system: Model development and numerical simulation. Chemical Engineering Journal 97, 47-67.

Lawrence, L. L. (1990). Probability and statistics for modern engineering, 2nd ed. New York: McGraw-Hill.
Lin, K.-P. \& Chen, M.-S. (2011). On the design and analysis of the privacy-preserving SVM classifier. IEEE Transaction on Knowledge and Data Engineering, 23(11).

Lin, K.-P. \& Chen, M.-S. (2008). Releasing the SVM classifier with privacy preservation. 2008 Eighth IEEE International Conference on Data Mining.

Liu, M., Zang, S., \& Zhou, D. (2005). Fast leak detection and location of gas pipelines based on an adaptive particle filter. International Journal of Applied Mathematics and Computer Science, 541-550.

Luyong, S., et al. (2010). Research of leak detection and exclusion system based on pressure differential discrimination method. IEEE.

Ming, W. \& Wei-qiang, W. (2006). Application of Wavelet to Detect Pipeline Leak Point. Proceedings of the Sixth International Conference on Intelligent Systems Design and Applications (ISDA'06).

Murvay, P.-S. \& Silea, I. (2012). A survey on gas leak detection and localization techniques. Journal of Loss Prevention in the Process Industries 25, 966-973.

Musbah, M. S. \& Zhu, X. (2007). Support vector machines for DS-UWB channel equalisation. Department of Electrical Engineering \& Electronics, University of Liverpool, UK, IEEE, pp. 524-527.

Rongsheng, G., Gongtian, S., \& Shifeng, L. (2002). An overview on the development of acoustic emission signal processing and analysis technique. Nondestructive Testing, 24(1), 23-28.

Sami, L. H.-T. \& Ezzeddine, H.-T. (2010). Leak detection of hydrogen-natural gas mixtures in pipes using the characteristics method of specified time intervals. Journal of Loss Prevention in the Process Industries 23, 637-645.

Takens, F. (1981). Detecting strange attractors in 
turbulence. In: D. Rand \& L. S. Young (eds.), Dynamical systems and turbulence. Lecture Notes Math., 898. Berlin: Springer-Verlag, pp. 366-382.

Tani Tetsuji, et al. (2006). Chaos information criteria to detect high-pressure gas leak in petroleum refining plant. SICE-ICASE International Joint Conference 2006, Oct. 18-21, in Bexco, Busan, Korea.

Tani, T., Nagasako, T., \& Lokibe, T. (2004). High pressure gas leak detection by a chaotic approach in a petroleum refining plant. Smart engineering system design. Neural Networks, Fuzzy Logic, Evolutionary Programming, Complex Systems and Artificial Life, 14, ASME, 889-904.

Tao, Y. \& Malshe, A. (2005). Theoretical investigation on hermeticity testing of MEMS packages based on MIL-STD-883E. Microelectron. Rel., 45(3-4), 559-566.

Tongtai Xu. (1997). Lost circulation prevention and plugging technology in drilling [M]. Beijing: Petroleum Industry, pp. 35-50.

Vaz Junior, C. A., et al. (2010). ARX modeling approach to leak detection and diagnosis. Journal of Loss Prevention in the Process Industries 23, 462-475.

Wang Xin, Liu Jianguo, Ma Weidong, et al. (2002). The progress of pipeline leak testing technolo- gy at home and abroad. Chemical Equipment \& Anticorrosion, 5, 40-44.

Yang Jiao, Yang Qingxin, Li Guanghai, \& Zhang Jingyan. (2006). Acoustic emission source identification technique for buried gas pipeline leak. IEEE.

Yang Jin, Wen Yumei, \& Li Ping. (2010). Approximate entropy-based leak detection using artificial neural network in water distribution pipelines. 2010 11th Int. Conf. Control, Automation, Robotics and Vision Singapore, 7-10th December.

Yunhu Lu, Mian Chen, Yan Jin, \& Hui Hui. (2011). Identification of leak zone pre-drilling based on fuzzy control. IEEE.

Zadeh, L. A. (1965). Fuzzy sets. Information and Control, 8, 338-353.

Zhang, L., Qin, X., Wang, Z., \& Liang, W. (2009). Designing a reliable leak detection system for West products pipeline. Journal of Loss Prevention in the Process Industries, 22, 981-989.

Zhi-Jie Zhou, et al. (2011). Bayesian reasoning approach based recursive algorithm for online updating belief rule based expert system of pipeline leak detection. Expert Systems with Applications 38, 3937-3943.

Zhiyin Gan, et al. (2008). Measurement of leak rate for MEMS vacuum packaging. IEEE. 